\title{
The Influence of Plant Hormones on Phospholipid Monolayer Stability
}

\author{
Barbara Gzyl-Malcher ${ }^{\mathrm{a}, *}$, Maria Filek ${ }^{\mathrm{b}, \mathrm{c}}$, Gerald Brezesinski ${ }^{\mathrm{d}}$, and Antje Fischer ${ }^{\mathrm{d}}$ \\ a Department of Physical Chemistry and Electrochemistry, Faculty of Chemistry, \\ Jagiellonian University, Ingardena 3, 30-060 Kraków, Poland. Fax: 0048126340515. \\ E-mail: gzyl@chemia.uj.edu.pl \\ b Institute of Biology, Pedagogical Academy, Podbrzezie 3, 31-054 Kraków, Poland \\ c Institute of Plant Physiology, Polish Academy of Sciences, Podłużna 3, 30-239 Kraków, \\ Poland \\ d Max Planck Institute of Colloids and Interfaces, D-14476 Golm/Potsdam, Germany \\ * Author for correspondence and reprint requests \\ Z. Naturforsch. 62 c, 55-60 (2007); received July 24/September 8, 2006
}

The influence of hormones in water subphase on the stability of monolayers built of phospholipid mixtures extracted from embryogenic (PLE) and nonembryogenic (PLNE) wheat calli was examined. Additionally, experiments on individual lipids, dipalmitoylphosphatidylcholine (DPPC) and dipalmitoylphosphatidic acid (DPPA), were performed. DPPC was chosen because it was the main phospholipid present in both calli. Negatively charged DPPA could mimic a negatively charged natural mixture of lipids. As hormones, auxins (IAA and 2,4-D), cytokinins (zeatin and kinetin) and zearalenone were chosen. The time of monolayer stability for PLNE calli was much longer than for PLE calli. Kinetics of monolayer stability of PLNE was similar to DPPA, whereas that of PLE was similar to DPPC. Generally, hormones increased the time after which the monolayer stability was reached and decreased the surface pressure. The greatest effect was observed for auxins (especially IAA), whereas cytokinins affected the monolayer stability to a lesser degree.

Key words: Phospholipid Monolayer, Auxins, Cytokinins

\section{Introduction}

Plant hormones control a wide range of plant growth and developmental processes, however, the molecular mechanisms of hormone action are still poorly understood (Davies, 1995). Within the last years, biochemical approaches have been concentrated on the identification of protein membrane hormone receptors (Jones, 1994; Ma, 1998; Hooley, 1999; Elrouby and Bureau, 2000; Dannies, 2001; Romanov, 2002). In spite of a growing number of studies, the functional role of hormones in signalling and transport remained unclear, especially as regards the time of hormone action-cell reaction. Since the majority of hormones bear a charge, they can be adsorbed to specific membrane lipid domains (Filek et al., 2002). Moreover, other hormone-like substances having a hydrophobic character were found (Meng et al., 1996; Fu et al., 2000). In the case of that kind of hormones, the interaction with the hydrophobic (fatty acids) part of membranes is possible.

A rapid response (within less than $1 \mathrm{~min}$ ) to hormone action at the outer face of the cell membrane reflects the modulation of the membrane ion transport (Venis et al., 1990). These data were received during studies of the electrophysiological response of plant protoplasts to hormones. Since the results of experiments on natural membranes are complex and difficult to interpret, it is necessary to conduct research on model systems, such as liposomes and monolayers (Bringezu and Brezesinski, 2001; Gambinossi et al., 2002; Popova et al., 2002; Latowski et al., 2003; Berglund et al., 2004; Filek et al., 2005; Gzyl and Paluch, 2005). From our electrophysiological experiments, zeta potential of protoplast membranes and phospholipid liposomes was stabilized within $5 \mathrm{~min}$ in the presence of hormones (Filek et al., 2002). In our monolayer experiments, it was found that hormones affect the isotherms of monolayers built from phospholipids extracted from natural plant cells at various stages of the competence to regeneration (nonembryogenic and embryogenic cultures) (Filek et al., 2003; Gzyl et al., 2003). The aim of the present study was to find how far those hormones can modify the monolayer stability under the maximum superficial density. On the basis of our earlier results we chose a surface pressure of $20 \mathrm{mN} / \mathrm{m}$, at which lipids extracted from embry- 
ogenic and nonembryogenic calli form a compact monolayer. Under these conditions we investigated the relaxation of phospholipid monolayers, with and without the influence of plant hormones, within a time limit of $30 \mathrm{~min}$ after the compression was stopped. The changes in relaxation of mixed phospholipid monolayers under hormone influence should be the results of hormone interactions with polar head groups of the lipids. Therefore, we also checked the hormone influence on relaxation of monolayers built from individual phospholipids (phosphatidylcholine and phosphatidic acid) having the same hydrophobic parts.

\section{Experimental}

Hormones [indole-3-acetic acid (IAA), 2,4-dichlorophenoxyacetic acid (2,4-D), zeatin, kinetin and zearalenone] as well as lipids [dipalmitoylphosphatidylcholine (DPPC) and dipalmitoylphosphatidic acid (DPPA)] were obtained from Sigma Chemical Co. Natural phospholipid fractions were isolated from winter wheat cells of calli cv. Kamila. Seedlings were vernalized at $5{ }^{\circ} \mathrm{C}$ with an $8 \mathrm{~h}$ photoperiod for 9 weeks. After that, the plants were planted in soil and grown in a glasshouse at $20 / 17^{\circ} \mathrm{C}$ (day/night) until the immature inflorescences appeared. The immature inflorescences were taken from the plants as explants to induce tissue culture. After sterilisation in $70 \%$ ethanol, the explants were cultured in the dark on modified Murashige and Skoog (MS) medium containing $1.14 \mu \mathrm{M} 2,4-\mathrm{D}$ as a source for nonembryogenic (NE) calli. For embryogenesis (E), 3month-old calli were transferred into MS medium without 2,4-D for 2 weeks. Phospholipids were extracted from the plasmalemma of NE and E cell cultures. Callus cells were filtered using a stainless net (mesh diameter was $120 \mu \mathrm{m}$ ) and homogenized at $4{ }^{\circ} \mathrm{C}$ in a solution containing $250 \mathrm{~mm}$ sucrose, $1 \mathrm{~mm}$ ethylenediaminetetraacetic acid (EDTA), $2.5 \mathrm{~mm}$ dithiothreitol (DTT), $1 \mathrm{~mm}$ phenylmethylsulfonyl fluoride (PMSF) and $10 \mathrm{~mm}$ tris(hydroxymethyl)aminomethane (Tris) buffer, adjusted to $\mathrm{pH} 7.5$ with $\mathrm{HCl}$. After two centrifugation steps at $10,000 \times g$ and $8,000 \times g$, the microsomal fraction was re-suspended in $5 \mathrm{~mm}$ potassium phosphate buffer ( $\mathrm{pH} 7.8$ ) containing $250 \mathrm{~mm}$ sucrose and $5 \mathrm{~mm} \mathrm{KCl}$ and added to a phase mixture consisting of $6.5 \%(\mathrm{w} / \mathrm{w})$ dextran T500, $6.5 \%$ $(\mathrm{w} / \mathrm{w})$ polyethyleneglycol (PEG) 4000, $5 \mathrm{~mm}$
$\mathrm{K}_{3} \mathrm{PO}_{4}$ ( $\mathrm{pH} 7.8$ ), $250 \mathrm{~mm}$ sucrose and $4 \mathrm{~mm} \mathrm{KCl}$. After a three-step phase partitioning, the upper phase (plasmalemma) was centrifuged at $100,000 \times g$ with $10 \mathrm{~mm}$ Tris/4-morpholineethanesulfonic acid buffer ( $\mathrm{pH} 7.4), 250 \mathrm{~mm}$ sucrose and $1 \mathrm{~mm}$ ethylenebis(oxyethylenenitrilo)tetraacetic acid (EGTA). The recovered plasmalemma fraction was extracted with a mixture of chloroform/ isopropanol $(1: 1 \mathrm{v} / \mathrm{v})$, and re-extracted with chloroform. The fractions of phospholipids, glycolipids and neutral lipids were isolated using adsorptive and distributive column chromatography on silica gel. The phospholipid (PL) fractions were mixed with chloroform and evaporated under an $\mathrm{N}_{2}$ atmosphere. They were freeze-dried and stored in the dark at $-20{ }^{\circ} \mathrm{C}$. The polar head group and fatty acid composition of the plasmalemma phospholipids were determined by thin-layer and gas chromatography, essentially described previously (Laggner et al., 2003a). The composition of the phospholipid mixture extracted from nonembryogenic calli (PLNE) was: a) hydrophobic tails: palmitic acid $(27 \%)$; stearic acid $(0.5 \%)$; oleic acid $(7 \%)$; linoleic acid $(50.5 \%)$; linolenic acid $(15 \%)$; b) hydrophilic head groups: phosphatidylcholine (33\%); phosphatidylethanolamine (31\%); phosphatidic acid (10\%); phosphatidyl glycerol (12\%); phosphatidylinositol (8\%); phosphatidylserine $(6 \%)$. The composition of the phospholipid mixture extracted from embryogenic calli (PLE) was: a) hydrophobic tails: palmitic acid (24\%); stearic acid $(0.5 \%)$; oleic acid $(2.5 \%)$; linoleic acid $(54 \%)$; linolenic acid (19\%); b) hydrophilic head groups: phosphatidylcholine (32\%); phosphatidylethanolamine (34\%); phosphatidic acid (13\%); phosphatidyl glycerol (10\%); phosphatidylinositol (6\%); phosphatidylserine $(5 \%)$. The spreading solvent was a chloroform/methanol $(4: 1 \mathrm{v} / \mathrm{v})$ mixture. Chloroform and methanol were purchased from Sigma Chemical Co. Bidistilled water was used as a subphase.

The surface pressure was determined by using a KSV 1000 balance apparatus (KSV Instruments Ltd, Helsinki, Finland). The sensitivity of the film pressure and area measurements were $\pm 0.01 \mathrm{mN} /$ $\mathrm{m}$ and $\pm 0.01 \AA^{2} /$ molecule, respectively. Each experiment was repeated at least twice. The subphase temperature $\left(20^{\circ} \mathrm{C}\right)$ was controlled thermostatically to $\pm 0.1{ }^{\circ} \mathrm{C}$ by circulating water. To measure the surface pressure relaxation, the spread monolayers were compressed to $20 \mathrm{mN} / \mathrm{m}$ (with a compression rate of $6 \mathrm{~mm} / \mathrm{min}$ ) and then 
allowed to rest at a constant area. For the experiments with phytohormones, their solutions in water $(1 \mu \mathrm{M})$ were used as a subphase. The stability limit was measured by observing the decay in the surface pressure as a function of time.

\section{Results and Discussion}

The monolayer relaxation experiments based on monitoring the surface pressure changes indicate that, for the DPPC monolayer (Fig. 1), a plateau was attained after $10.4 \mathrm{~min}$. In Table I the values of the time after which the plateau is reached $(t)$, the surface pressure of the plateau $\left(\pi_{\text {plateau }}\right)$ and the surface pressure values after 10 and 20 minutes $\left(\pi_{10}\right.$ and $\pi_{20}$, respectively) are shown. The zwitterionic character of the polar head of DPPC may favour the interactions between these molecules as well as between them and water, causing the monolayer stabilization. The DPPA monolayer behaves obviously differently (Fig. 2): a constant $\pi$ value was not reached before $26.5 \mathrm{~min}$, what may be attributed to processes related to monolayer reorganization within that time. DPPA is a phospholipid bearing a negative charge causing the intermolecular repulsion or specific domain formation with water molecules. Formation of these domains may be the reason of the monolayer reorganization and surface pressure lowering. Indeed, for that monolayer the largest surface pressure drop was observed (about $1 \mathrm{mN} / \mathrm{m}$ ). Considering the monolayers of natural mixture, a lower relaxation time was recorded for PLE (5.1 min) (Fig. 3) than for PLNE (21.3 min) (Fig. 4). A greater percentage content of PA in the PLE mixture would suggest the enhanced instability of that monolayer in comparison with PLNE. However, in lipid mixtures, interactions between different lipids may compensate the interactions between the same lipids. Moreover, PLE contain more unsaturated chains than PLNE. Unsaturated fatty acids participating in the domain formation may stabilize these domains to a higher degree by the steric limitation. PLE and PLNE monolayers show a relaxation behaviour similar to DPPC and DPPA, respectively.

Phytohormones affect the surface pressure decrease observed in lipid monolayer relaxation. The greatest decay of surface pressure was recorded in the presence of auxins in all systems, the lowest in the presence of cytokinins. Therefore, we can conclude that negatively charged hormones (IAA and 2,4-D) affect the monolayer disorganization to the highest degree, whereas the effect of positively charged compounds (kinetin and zeatin) is significantly lower. The influence of zearalenone is intermediate between that of auxins and that of cytokinins, with the exception of PLNE monolayers where zearalenone exerts the smallest effect.

Considering hormone influence on the stability of individual lipid monolayers, a smaller effect was observed for a DPPA than for a DPPC monomolecular layer (Figs. 1 and 2). Therefore, we can conclude that hormone penetration into the less stable DPPA monolayer is easier and it disturbs the surface equilibrium to a lesser degree. The largest effect observed for negatively charged hor-

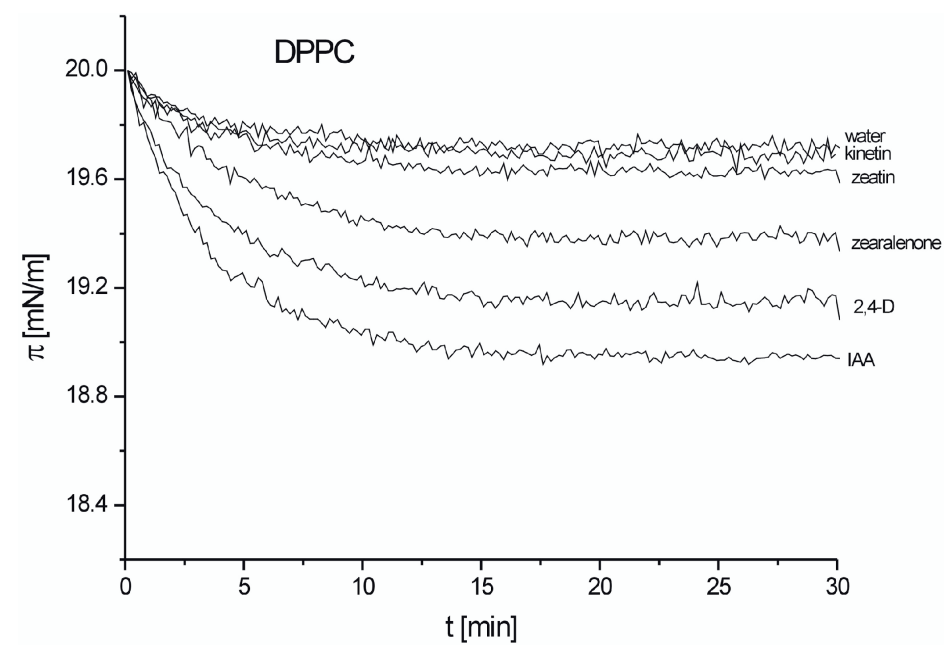

Fig. 1. Relaxation of DPPC monolayers on pure water and water with hormones. 
Table I. Characteristic parameters for relaxation of lipid monolayers on different subphases at constant surface pressure of $20 \mathrm{mN} / \mathrm{m}$ and $20^{\circ} \mathrm{C}$. $\pi_{10}$ and $\pi_{20}$, surface pressure values after 10 minutes and 20 minutes; $t$, time after which the plateau is reached; $\pi_{\text {plateau }}$, surface pressure of the plateau; hormone concentration: $1 \mu \mathrm{M}$.

\begin{tabular}{llcccc}
\hline Phospholipids & Hormones & $t[\mathrm{~min}]$ & $\pi_{10}[\mathrm{mN} / \mathrm{m}]$ & $\pi_{20}[\mathrm{mN} / \mathrm{m}]$ & $\pi_{\text {plateau }}[\mathrm{mN} / \mathrm{m}]$ \\
\hline DPPC & Water & $10.4 \pm 0.2$ & $19.75 \pm 0.02$ & $19.72 \pm 0.02$ & $19.72 \pm 0.01$ \\
& Zeatin & $12.6 \pm 0.2$ & $19.68 \pm 0.02$ & $19.63 \pm 0.01$ & $19.63 \pm 0.02$ \\
& Kinetin & $12.7 \pm 0.1$ & $19.73 \pm 0.01$ & $19.69 \pm 0.02$ & $19.69 \pm 0.02$ \\
& IAA & $13.7 \pm 0.2$ & $19.03 \pm 0.03$ & $18.95 \pm 0.02$ & $18.95 \pm 0.01$ \\
& 2,4-D & $13.4 \pm 0.2$ & $19.24 \pm 0.03$ & $19.16 \pm 0.02$ & $19.16 \pm 0.02$ \\
DPPA & Zearalenone & $13.5 \pm 0.3$ & $19.47 \pm 0.03$ & $19.38 \pm 0.01$ & $19.38 \pm 0.01$ \\
& Water & $26.5 \pm 0.3$ & $19.37 \pm 0.02$ & $19.14 \pm 0.02$ & $19.07 \pm 0.02$ \\
& Zeatin & $26.9 \pm 0.2$ & $19.21 \pm 0.03$ & $18.91 \pm 0.02$ & $18.81 \pm 0.02$ \\
& Kinetin & $26.4 \pm 0.2$ & $19.28 \pm 0.03$ & $19.00 \pm 0.02$ & $18.92 \pm 0.02$ \\
PLE & IAA & $27.4 \pm 0.2$ & $19.03 \pm 0.03$ & $18.73 \pm 0.03$ & $18.61 \pm 0.03$ \\
& 2,4-D & $26.4 \pm 0.3$ & $19.13 \pm 0.03$ & $18.79 \pm 0.03$ & $18.67 \pm 0.02$ \\
& Zearalenone & $27.8 \pm 0.2$ & $19.21 \pm 0.03$ & $18.91 \pm 0.02$ & $18.80 \pm 0.02$ \\
& Water & $5.1 \pm 0.1$ & $19.84 \pm 0.01$ & $19.82 \pm 0.01$ & $19.81 \pm 0.03$ \\
& Zeatin & $5.9 \pm 0.2$ & $19.73 \pm 0.02$ & $19.69 \pm 0.02$ & $19.69 \pm 0.02$ \\
KLNE & Kinetin & $19.65 \pm 0.02$ & $19.61 \pm 0.01$ & $19.60 \pm 0.01$ \\
& IAA & $21.3 \pm 0.2$ & $19.36 \pm 0.02$ & $19.15 \pm 0.02$ & $19.10 \pm 0.02$ \\
& 2,4-D & $11.4 \pm 0.1$ & $19.50 \pm 0.02$ & $19.37 \pm 0.02$ & $19.35 \pm 0.02$ \\
& Zearalenone & $10.4 \pm 0.2$ & $19.68 \pm 0.01$ & $19.60 \pm 0.01$ & $19.59 \pm 0.02$ \\
& Water & $21.3 \pm 0.3$ & $19.43 \pm 0.02$ & $19.30 \pm 0.01$ & $19.26 \pm 0.01$ \\
& Zeatin & $20.7 \pm 0.3$ & $19.17 \pm 0.03$ & $19.05 \pm 0.01$ & $19.03 \pm 0.03$ \\
& Kinetin & $23.4 \pm 0.2$ & $19.33 \pm 0.02$ & $19.17 \pm 0.03$ & $19.11 \pm 0.03$ \\
& IAA & $21.6 \pm 0.2$ & $19.20 \pm 0.02$ & $19.04 \pm 0.01$ & $19.01 \pm 0.02$ \\
& 2,4-D & $21.0 \pm 0.3$ & $19.21 \pm 0.03$ & $19.04 \pm 0.01$ & $19.02 \pm 0.02$ \\
& Zearalenone & $23.3 \pm 0.2$ & $19.37 \pm 0.03$ & $19.25 \pm 0.02$ & $19.18 \pm 0.02$ \\
\hline
\end{tabular}

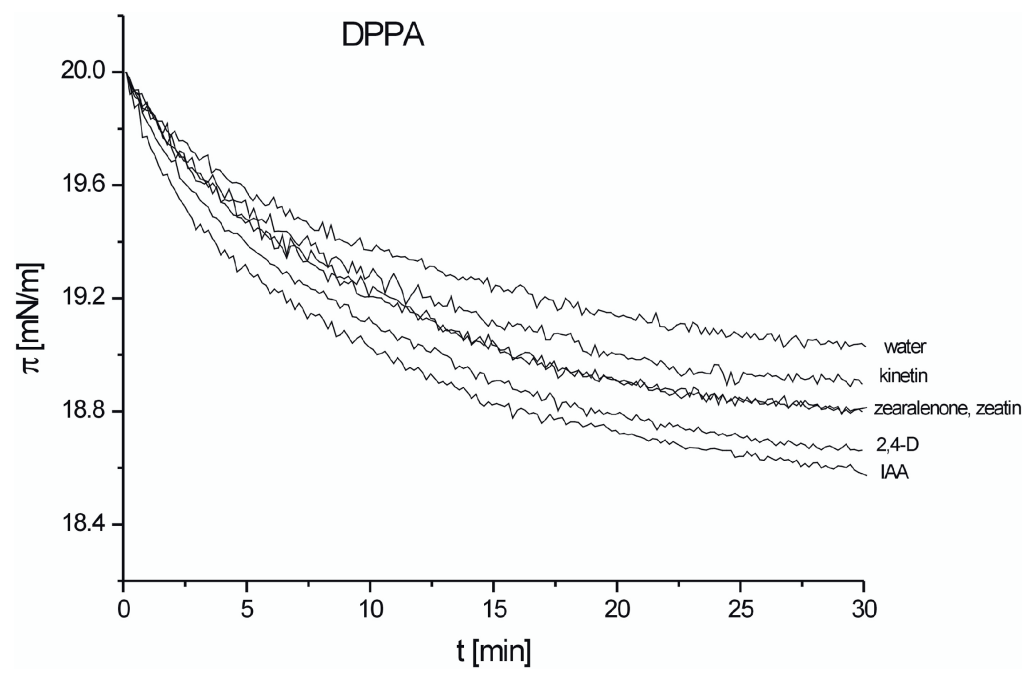

Fig. 2. Relaxation of DPPA monolayers on pure water and water with hormones. mones may confirm a previous suggestion, that the instability of a DPPA monolayer is connected with interactions between negatively charged molecules. However, auxins exert influence also on the relaxation process of the DPPC monolayer, although that lipid bears not only negative but also positive charges in the polar part. Therefore, one can assume that the presence of a negative charge in lipid molecules forming the monomolecular layer determines the instability of that monolayer in the presence of auxines. The steric properties of hormone molecules may be responsible for the 


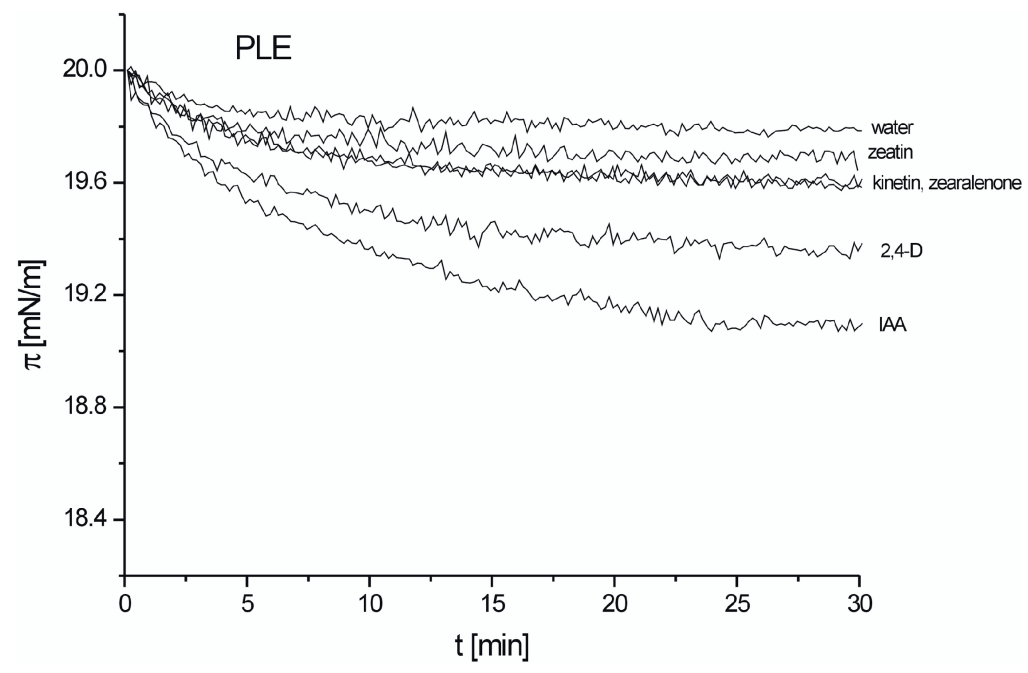

Fig. 3. Relaxation of PLE monolayers on pure water and water with hormones.

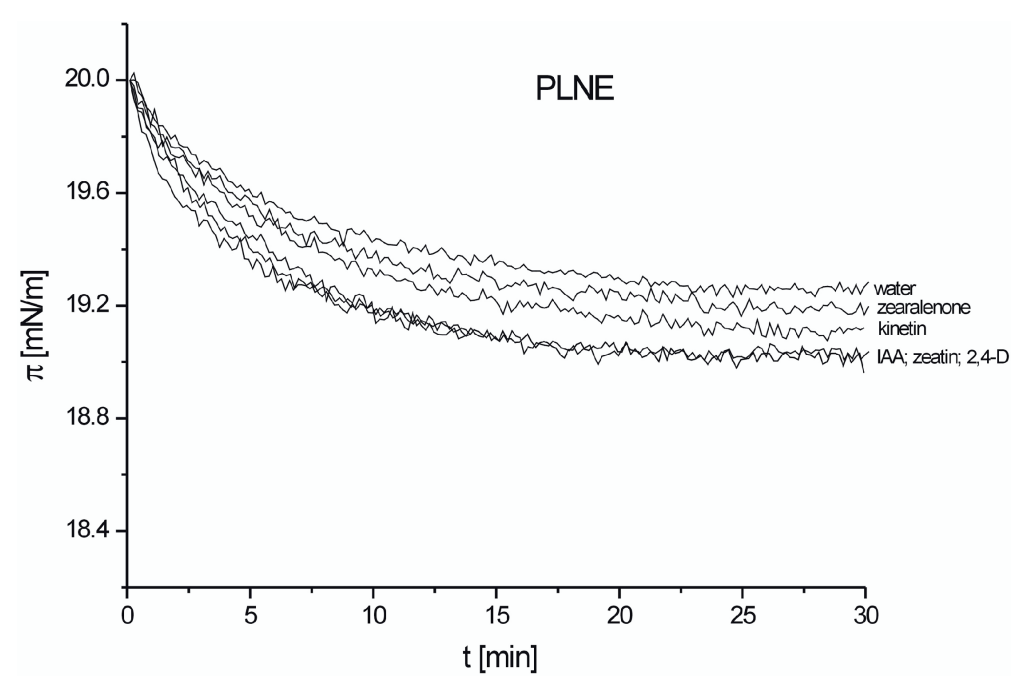

Fig. 4. Relaxation of PLNE monolayers on pure water and water with hormones. differences in the activity of hormones belonging to the same classes. The greater influence of IAA compared to 2,4-D can result from the presence of the additional ring in the IAA molecule, which can hinder the monolayer stability achievement. On the other hand, the smaller differences between the effect of zeatin and kinetin can suggest that the presence of a purine ring in both molecules is the main steric parameter determining the interactions with the lipid monolayer. As regards lipid mixture monolayers, one can notice the smaller hormone influence on the PLNE monolayer (Figs. 3 and 4) in comparison to the PLE system. The possible reason for that behaviour may be the same as in the case of DPPA and DPPC systems, i.e. the lower stability of PLNE. For PLE, analogously to DPPC monolayers, the highest instability was observed in the presence of auxins (especially IAA), whereas cytokinins exert the smallest effect. In the case of PLNE the order of the hormone influence on the relaxation process is not exactly the same as in the DPPA system: zearalenone and kinetin exert the smallest influence, whereas the effect of zeatin is similar to that of auxins and it changes the relaxation time and decreases the surface pressure at the plateau to the highest degree. Smaller hormone influence on the destabilization of a PLNE monolayer in comparison with a PLE monolayer may be responsible for smaller changes in surface parameters of PLNE observed in the 
presence of hormones (Gzyl et al., 2003; Laggner et al., 2003b).

To conclude, we found that comparing the results obtained for monolayers of single lipids and mixtures, the relaxation behaviour of a PLE monolayer was similar to DPPC, and the one of PLNE was similar to DPPA. Hormones affected the monolayer stability, increasing the time after which the constant surface pressure was reached and decreasing the surface pressure values. The orders of hormone influence on PLE and DPPC

Berglund A. H., Larsson K. E., and Liljenberg C. S. (2004), Permeability behaviour of lipid vesicles prepared from plant plasma membranes - impact of compositional changes. Biochim. Biophys. Acta 1682, $11-17$.

Bringezu F. and Brezesinski (2001), Chemically modified lipids - a suitable tool to study molecular interactions in model systems. Coll Surf. A 183-185, 391-401.

Dannies P. S. (2001), A serum prolactin-binding protein: implications for growth hormone. Trends Endocrin. Metab. 12, 427-428.

Davies P. J. (1995), Plant Hormones: Physiology, Biochemistry and Molecular Biology. Kluwer Academic Publishers, Dordrecht, The Netherlands, pp. 1-38.

Elrouby N. and Bureau T. E. (2000), Molecular characterization of the Abp1 5'-flanking region in maize and the teosintes. Plant Physiol. 124, 369-378.

Filek M., Zębala M., and Szechyńska-Hebda M. (2002), The influence of phytohormones on zeta potential and electrokinetic charges of winter wheat cells. Z. Naturforsch. 57c, 696-704.

Filek M., Gzyl B., and Dudek A. (2003), The influence of phytohormones on the properties of wheat phospholipid monolayers at the water-air interface. Cell. Mol. Biol. 8, 713-726.

Filek M., Gzyl B., Laggner P., and Kriechbaum M. (2005), Effect of indole-3-acetic acid on surface properties of the wheat plastid lipids. J. Plant Physiol. 162, $245-252$.

Fu Y.-F., Han Y.-Z., Zhao D.-G., and Meng F.-J. (2000), Zearalenone and flower bud formation in thin-cell layers of Nicotiana tabacum L. Plant Growth Regul. 30, 271-274.

Gambinossi F., Puggeli M., and Gabrielli G. (2002), Enzymatic hydrolysis reaction of phospholipids in monolayers. Coll. Surf. B. 23, 273-281.

Gzyl B. and Paluch M. (2005), Mixed monolayers of dipalmitoyl phosphatidylcholine and ethyl palmitate at monolayers were similar, i.e. the smallest effects were observed for cytokinins, the greatest for auxins. However, in the case of PLNE, the influence of hormones on the relaxation process was different than in the DPPA monolayer. Therefore, on the basis of research conducted on the individual lipids one can not draw conclusions about the interactions in the natural lipid mixture, because the specificity associated with the domain formation may determine intermolecular interactions.

the air/water interface. Appl. Surface Sci. 246, 356361 .

Gzyl B., Filek M., and Dudek A. (2003), Influence of temperature on phytohormone interactions with monolayers obtained from phospholipids of wheat calli. Z. Naturforsch. 59c, 60-64.

Hooley R. (1999), A role for G proteins in plant hormone signaling? Plant Physiol. Biochem. 37, 393-402.

Jones A. M. (1994), Auxin-binding proteins. Annu. Rev. Plant Physiol. Plant Mol. Biol. 45, 393-420.

Laggner P., Filek M., Kriechbaum M., Marcińska I., and Szechyńska-Hebda M. (2003a), X-Ray structure investigations of winter wheat membrane systems. I. Influence of phytohormones on phospholipid orientation in non- and embryogenic cells. Plant Sci. 165, $265-270$.

Laggner P., Filek M., Kriechbaum M., Marcińska I., and Szechyńska-Hebda M. (2003b), X-Ray structure investigations of winter wheat membrane systems. II. Effect of phytohormones on structural properties of mixed phospholipid-sterols membranes. Plant Sci. 165, 271-275.

Latowski D., Kostecka-Gugała A., and Strzałka K. (2003), Effect of the temperature on violaxanthin deepoxidation: comparison of the in vivo and model systems. Russian J. Plant Physiol. 50, 173-177.

Ma H. (1998), A serpentine receptor surfaces in Arabidopsis. Trends Plant Sci. 3, 248-250.

Meng F. J., Han Y. Z., Fu Y. F., and Guo F. L. (1996), Zearalenone in higher plants. Flowering Newslett. 22, $54-57$.

Popova A. V., Heyer A. G., and Hincha D. K. (2002), Differential destabilization of membranes by tryptophan and phenylalanine during freezing: the roles of lipid composition and membrane fusion. Biochim. Biophys. Acta 1561, 109-118.

Romanov G. A. (2002), The phytohormone receptors. Russ. J. Plant Physiol. 49, 552-560.

Venis M. A., Thomas E. W., Barbier-Brygoo H., Ephritikine G., and Guen J. (1990), Impermeant auxin analogues have auxin activity. Planta 182, 232-235. 